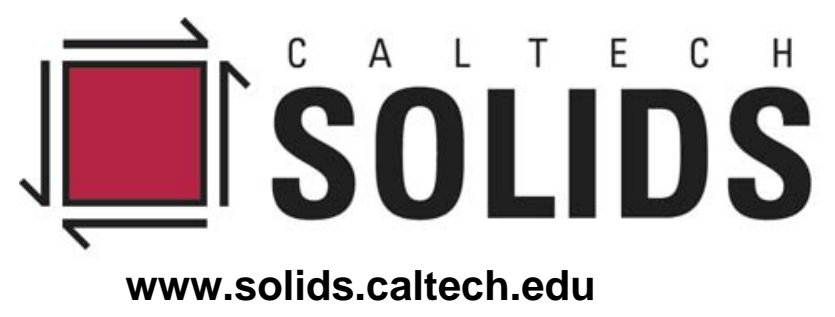

\title{
A SHARP INTERFACE MODEL FOR THE PROPAGATION OF MARTENSITIC PHASE BOUNDARIES
}

P.W. Dondl and K. Bhattacharya

November 24, 2008 


\title{
A sharp interface model for the propagation of martensitic phase boundaries
}

\author{
Patrick W. Dondl \\ Max-Planck-Institute for Mathematics in the Sciences, Leipzig, Germany \\ Email: pwd@mis.mpg.de \\ and \\ Kaushik Bhattacharya \\ California Institute of Technology, Pasadena, USA \\ Email: bhatta@caltech.edu
}

November 24, 2008

\begin{abstract}
A model for the quasistatic evolution of martensitic phase boundaries is presented. The model is essentially the gradient flow of an energy that can contains elastic energy due to the underlying change in crystal structure in the course of the phase transformation and surface energy penalizing the area of the phase boundary. This leads to a free boundary problem with a nonlocal velocity that arises from the coupling to the elasticity equation. We show existence of solutions under a technical convergence condition using an implicit time-discretization.
\end{abstract}

\section{Introduction}

The martensitic phase transformation is a solid-to-solid phase transformation characterized by a change in crystal structure and the absence of any diffusion or rearrangement of the lattice. It has long been recognized that a continuum thermoelasticity model of such phase transitions gives rise to piece-wise smooth solutions or non-classical solutions that are interpreted to be the microstructure that is commonly observed in materials that undergo this transition [9, 6, 7]. This paper is concerned with global solvability of a sharp interface model for the evolution of martensitic phase boundaries. Specifically, we start with a model that is the quasistatic analog of those developed by Abeyaratne and Knowles [1, 2], and develop a mathematical theory that builds on the work of Luckhaus and Sturzenhecker [11] who studied mean curvature flow.

Consider a body occupying a region $\Omega \subset \mathbb{R}^{n}, n \in \mathbb{N}$ with a phase boundary $\Gamma$ separating two phases that occupy the regions $E$ and $\Omega \backslash E$ at some given time $t$ as shown in Figure 1 . The body may also contain non-transforming regions or inclusions $A_{i}$. The total energy of the body is given as

$$
\begin{aligned}
\mathcal{F}(E) & =\mathcal{F}_{\text {elastic }}(E)+\mathcal{F}_{\text {surface }}(E) \\
& =\min _{u} \int_{\Omega} \frac{1}{2}\left|\nabla u(x)-\xi_{E}(x)\right|^{2} \mathrm{~d} x+\int_{\Omega}\left|\nabla \chi_{E}\right| \mathrm{d} x
\end{aligned}
$$

where

$$
\xi_{E}(x)=\left\{\begin{array}{lll}
\xi^{+} & \text {for } & x \in \Omega \backslash(E \cup A) \\
\xi^{-} & \text {for } & x \in E \backslash A \\
\xi^{A} & \text { for } & x \in A
\end{array}\right.
$$

The first term in (1) represents the elastic energy and the second the interfacial energy, $|\cdot|$ denotes the Euclidean norm. For simplicity we take $u$ to be a scalar, though this is not necessary as we discuss later. Briefly, since the phases have different crystal structures, the strains take the values $\xi^{+}$and $\xi^{-}$in the 


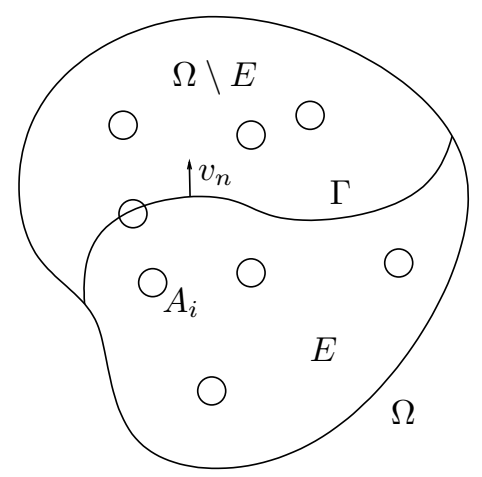

Figure 1: Domain occupied by a phase transforming elastic body

two phases in the absence of any stress. Further, the non-transforming particles take the strain $\xi^{A}$ in the absence of any stress. The first integral penalizes deviations in strain from these preferred values. Importantly, we assume that the modulus is uniform or independent of phase. We minimize this energy over all possible displacements subject to appropriate boundary conditions to obtain the elastic energy. The interfacial energy penalizes the area of the phase boundary.

We are interested in the evolution of the phase boundary $\Gamma$. We postulate that its evolution is determined by the gradient flow of the energy (1); in other words, we postulate that the rate of change of the position of the boundary is proportional to the variation in total energy with position. To be precise, let us assume that we have a propagating phase boundary $\Gamma(t)$. Then it is possible to show that the rate of change of energy is given by (see Abeyaratne and Knowles [1])

$$
\frac{\mathrm{d}}{\mathrm{d} t} \mathcal{F}=-\int_{\Gamma}(\llbracket W \rrbracket-\langle\sigma\rangle \llbracket \nabla u \rrbracket-\kappa) v_{n} \mathrm{~d} x_{\Gamma}
$$

where $W=\frac{1}{2}\left|\nabla u-\xi_{E}\right|^{2}$ is the elastic energy density, and $\sigma=\nabla u-\xi_{E}$ is the stress, and $\llbracket \cdot \rrbracket$ and $<\cdot>$ denote the jump and average of some quantity across the boundary respectively. Also, $u$ is the minimizer of the elastic energy and satisfies

$$
\operatorname{div}\left(\nabla u-\xi_{E}\right)=0
$$

along with boundary conditions. Therefore its derivatives may be discontinuous across surfaces where $\xi_{E}$ is discontinuous. Following Abeyaratne and Knowles [1], we identify the term in the parenthesis in the integrand above as the thermodynamic driving force

$$
f=\llbracket W \rrbracket-\langle\sigma\rangle \llbracket \nabla u \rrbracket-\kappa
$$

and postulate that the evolution of the phase boundary follows the equation

$$
v_{n}=f=\llbracket W \rrbracket-\langle\sigma\rangle \llbracket \nabla u \rrbracket-\kappa .
$$

Equation (6) defines a free-boundary problem that is the focus of this paper. The normal velocity of the boundary depends not only on position, but also on the solution of an auxiliary partial differential equation 4. This makes the free-boundary problem non-local. We provide a precise statement of the weak formulation of this problem in Section 2. A finite time discretization scheme is introduced and the convergence of the scheme is proved in Section 3 (Theorem 3.9). We prove in Section 4 that the limit of the time discrete solution as the time-step goes to zero satisfies the free boundary problem in the weak sense under the technical condition that there is no loss of surface energy (Theorem 4.3). The proofs follow Luckhaus and Sturzenhecker [11], though we have to address difficulties that arise from a lack of smoothness of the elastic strain fields. Specifically, solutions of (4) are not Lipschitz and thus the driving force is not bounded in $L^{\infty}$. We overcome this by mollifying the transformation strain $\xi_{E}$ and then take a careful double-limit.

To our knowledge, this is the first attempt towards a mathematically rigorous treatment of the evolution of martensitic phase boundaries under linear or viscous kinetics (6). We note that similar nonlocal free boundary problems have been studied in the context of gradient flows in metric spaces [5] and rate-independent processes [12]. The main mathematical difference is that a rate independent process, i.e., a gradient flow with respect to the $L^{1}$ norm (or any other gradient flow in a metric space) automatically 
yields better compactness properties for a time-discrete approximation. In this work, we have to make a density estimate by Luckhaus and Sturzenhecker applicable to our nonlocal case (Lemma 3.6).

In a separate paper, we examine the role of the non-transforming precipitates in some detail [8]. We introduce a shallow interface approximation, and show in this approximation that the effective motion of a phase boundary propagating locally with a linear or viscous kinetics in a periodic heterogeneous medium has a stick-slip behavior. In other words, there is a critical macroscopic driving force below which the interface is macroscopically stuck and the effective velocity grows singularly beyond. Thus, microscopic linear kinetics leads to macroscopic rate-independent kinetics (at least at small velocities) in the presence of heterogeneities.

The model presented here is written in terms of a scalar displacement, though this is not necessary. The proofs hold for vector displacements as well. Further, the results also holds for the problem of geometrically linear elasticity where the strain is the symmetrized displacement gradient by invoking the Korn's inequality in order to render the problem of finding the displacement elliptic. This ensures that the regularity estimates for the displacement gradient are still valid.

\section{Statement of the free boundary problem}

Given an initial phase distribution characterized by a set of finite perimeter $E_{0} \subset \Omega$, contained within the domain $\Omega$ with $C^{2}$ boundary, the free boundary problem, for a fixed final time $T>0$, can be posed in the weak form as follows on the time-space domain $\Omega_{T}=[0, T] \times \Omega$.

(P) Find functions $\chi: \Omega_{T} \rightarrow\{0,1\} \in L^{\infty}([0, T] ; B V(\Omega)), v: \Omega_{T} \rightarrow \mathbb{R} \in L^{1}\left([0, T] ; L^{1}(|\nabla \chi(t)|)\right)$, and $u: \Omega_{T} \rightarrow \mathbb{R} \in L^{2}([0, T] ; H(\Omega))$, such that

$$
\begin{array}{ll} 
& \int_{\Omega_{T}}\left(\operatorname{div} \zeta-\frac{\nabla \chi}{|\nabla \chi|} \nabla \zeta \frac{\nabla \chi}{|\nabla \chi|}\right)|\nabla \chi|+ \\
& \int_{\Omega_{T}}(W \cdot I d-\nabla u \otimes \sigma) \nabla \zeta+\int_{\Omega_{T}} v \zeta \nabla \chi=0 \\
\text { and } \quad & \text { for all } \zeta \in C^{\infty}\left(\overline{\Omega_{T}}, \mathbb{R}^{n}\right), \zeta=0 \text { on } \partial \Omega \times(0, T), \\
& \int_{\Omega_{T}} \chi \partial_{t} \xi+\int_{\Omega} \chi_{E_{0}} \xi(0)=-\int_{\Omega_{T}} v \xi|\nabla \chi| \\
& \text { for all } \xi \in C^{\infty}\left(\overline{\Omega_{T}}, \mathbb{R}^{n}\right), \xi=0 \text { on } \partial \Omega \times(0, T), \\
& \text { and } \xi(T)=0,
\end{array}
$$

as well as

$$
\begin{aligned}
& \int_{\Omega_{T}}\left(\nabla u-\xi_{E}\right) \nabla \phi=0 \\
& \text { for all } \phi \in C^{\infty}([0, T] ; H(\Omega)) .
\end{aligned}
$$

We consider $u$ in the space $H=\left\{u \in H^{1}(\Omega)\right.$, together with boundary conditions $\}$. Its dual space is denoted by $H^{\prime}$. The possible boundary conditions include Dirichlet as well as Neumann boundary conditions, together with a condition on the average of $u$ in order to make the elliptic problem wellposed. In fact, any boundary condition such that $\|\nabla u\|_{L^{\infty}}<c\|f\|_{L^{\infty}}$ holds for solutions of $\triangle u=f$ is admissable for the problem.

In order to see that a smooth solution of (7)-(9) indeed solves the original problem (6), consider, at a certain time $t \in(0, T)$, a phase boundary $\Gamma$ of class $C^{2}$. For the curvature term, first note that, by the surface divergence theorem, we have

$$
\int_{\Gamma} \operatorname{div}_{\Gamma} P \zeta=0
$$


where $P \zeta$ is the projection of $\zeta$ onto the tangent space of $\Gamma$. Now, by direct calculation, we have

$$
\begin{aligned}
0= & \int_{\Gamma} \partial_{k}\left(\zeta_{i}-\zeta_{j} n_{j} n_{i}\right) \cdot\left(\delta_{i k}-n_{i} n_{k}\right) \\
= & \int_{\Gamma} \zeta_{i, k} \cdot\left(\delta_{i k}-n_{i} n_{k}\right)-\int_{\Gamma} \zeta_{j} n_{j} \partial_{k} n_{i} \cdot\left(\delta_{i k}-n_{i} n_{k}\right) \\
& -\int_{\Gamma} \partial_{k}\left(\zeta_{j} n_{j}\right) n_{i} \cdot\left(\delta_{i k}-n_{i} n_{k}\right) \\
= & \int_{\Omega}\left(\operatorname{div} \zeta-\frac{\nabla \chi}{|\nabla \chi|} \nabla \zeta \frac{\nabla \chi}{|\nabla \chi|}\right)|\nabla \chi| \\
& -\int_{\Omega} \operatorname{div}{ }_{\Gamma} n \zeta \nabla \chi \\
& -\int_{\Gamma} \partial_{i}\left(\zeta_{j} n_{j}\right) n_{i}-\partial_{k}\left(\zeta_{j} n_{j}\right) n_{k} \\
= & \int_{\Omega}\left(\operatorname{div} \zeta-\frac{\nabla \chi}{|\nabla \chi|} \nabla \zeta \frac{\nabla \chi}{|\nabla \chi|}\right)|\nabla \chi| \\
+ & \int_{\Omega} \kappa \zeta \nabla \chi,
\end{aligned}
$$

where we are summing over repeated indices. We have also used the fact that - since the phase boundary is smooth - one can extend the function $n$ (the normal to the hypersurface), to a neighborhood of $\Gamma$.

For the nonlocal part of equation (7), first note that equation (9) ensures that $\operatorname{div} \sigma=0$ on $E$ and $\Omega \backslash E$, and $\llbracket \sigma \cdot n \rrbracket=0$ on the phase boundary $\Gamma$. Therefore the term involving derivatives of $\sigma$ that one obtains after integrating by parts vanishes. Now one can calculate

$$
\begin{aligned}
& \int_{\Omega}(W \cdot I d-\nabla u \otimes \sigma) \nabla \zeta \\
= & \int_{\Omega}(\llbracket W \rrbracket-\llbracket \nabla u \rrbracket\langle\sigma\rangle) \zeta \nabla \chi \\
& +\int_{\Omega \backslash \Gamma}\left(\frac{\partial W}{\partial \nabla u} \cdot \nabla^{2} u-\nabla^{2} u \cdot \sigma\right) \cdot \zeta .
\end{aligned}
$$

Here, we have split the divergence of the energy-momentum tensor up into a part that lives on the phase boundary, and a term that lives on the smooth part of the domain. The latter term vanishes, because here the energy density only depends on $\nabla u$, and not explicitly on the position.

Putting together the two calculations, we get

$$
\int_{\Omega}(-\kappa+\llbracket W \rrbracket-\llbracket \nabla u \rrbracket\langle\sigma\rangle-v) \zeta \nabla \chi=0,
$$

for all $\zeta \in C_{0}^{\infty}(\Omega)$, which yields $v=f$ on $\Gamma$. For a smoothly evolving phase boundary $\Gamma$, equation (8) is the definition of the normal velocity $v$ as the distributional time derivative of the evolving set.

\section{The time discrete approximation}

\subsection{Introduction to the finite time discretization scheme}

For a fixed time-step $h$, and a set of finite perimeter $E_{0}$, consider the following minimization problem. Find a set $E$, minimizing the energy

$$
\mathcal{F}(E)=\mathcal{F}_{\text {elastic }}(E)+\mathcal{F}_{\text {surface }}(E)+\int_{E \Delta E_{0}} \operatorname{dist}\left(x, \partial E_{0}\right) \mathrm{d} x .
$$

After proving that such a minimizer always exists, one can iteratively apply the minimization scheme and define an approximate solution as an in-time piecewise constant interpolation between the minimizers for each time step. The 'distance' term (an integral over the symmetric set difference of $E$ and $E_{0}$, denoted here by $E \triangle E_{0}$ ) in the energy is chosen such that its variation, for small $h$, is approximately the normal velocity of the phase boundary. This is the approach taken in $[3,11]$ for the related problem of 
a curvature flow. The second task is then to show convergence of the approximate solutions, as $h \rightarrow 0$, to a suitable solution of the original problem.

Since the sequence of piecewise constant approximate solutions is a sequence of characteristic functions on the space-time domain $\Omega \times[0, T]$, it naturally converges weakly* in $L^{\infty}$. It is however necessary to establish strong convergence here so that the resulting limit is again a characteristic function and one can view the problem as a set evolution. In order to do this, it is necessary to bound the total variation of the sequence of solutions uniformly both in space and in time. The uniform bound in space will be obtained directly from the uniform bound on the perimeter of the solutions. In order to derive the uniform bound in time, a density estimate on minimizing sets of the time-step problem is used in [11]. This estimate requires the forcing on the phase boundary to be uniformly bounded in $L^{\infty}$. Stress concentrations in the elasticity case, however, prevent its direct use. In order to overcome this difficulty, we regularize the elastic energy density by mollifying the transformation strain. The size $\varepsilon$ of the mollifier can then vanish in the right relation with the time-step $h$ and one can still establish strong convergence of the solution. This is detailed in the following section, where we use many similar ideas as [11].

\subsection{Local estimates on the elastic energy}

In order to prove compactness of the time discrete solutions later, the aforementioned stress concentrations must only occur in a very controlled manner. To this end, we regularize the transformation strain and will let the regularization tend to zero together with the time step in an appropriate way.

We first therefore define the mollified transformation strain

$$
\xi_{E}^{\varepsilon}(x)=\left(\eta^{\varepsilon} * \xi_{E}\right)(x)=\int_{\Omega} \eta^{\varepsilon}(x-y) \xi_{E}(y) \mathrm{d} y .
$$

Consider now the elastic energy

$$
\mathcal{F}_{\text {elastic }}(E)=\min _{u \in H} \int_{\Omega} \frac{1}{2}\left|\nabla u-\xi_{E}\right|^{2}
$$

and also the mollified elastic energy

$$
\mathcal{F}_{\text {elastic }}^{\varepsilon}(E)=\min _{u^{\varepsilon} \in H} \int_{\Omega} \frac{1}{2}\left|\nabla u^{\varepsilon}-\xi_{E}^{\varepsilon}\right|^{2} .
$$

It is clear from the theory of elliptic equations that such a minimizer always exists, since the transformation strain is a function in $L^{2}$. One can then derive the following a priori bounds.

Lemma 3.1. There exist $\varepsilon_{0}>0$ and a constant $M$ independent of $E$ and $\varepsilon$ such that, for $\varepsilon<\varepsilon_{0}$, the following hold.

i. $0<\mathcal{F}_{\text {elastic }}(E)<M$ and $0<\mathcal{F}_{\text {elastic }}^{\varepsilon}(E)<M$.

ii. For $u$, $u^{\varepsilon}$ attaining the minimum in $\mathcal{F}_{\text {elastic }}(E)$ and $\mathcal{F}_{\text {elastic }}^{\varepsilon}(E)$, respectively, we find $\|\nabla u\|_{L^{2}}<M$ and $\left\|\nabla u^{\varepsilon}\right\|_{L^{2}}<M$. iii. $\left\|\nabla u^{\varepsilon}\right\|_{L^{\infty}}<\frac{M}{\varepsilon^{n}}$.

Proof. $i$. Consider the test function $u=0$ on $\Omega$. Then $\int_{\Omega}\left|\xi_{E}\right|^{2}<\operatorname{m}(\Omega) \max \left(\xi^{ \pm}\right)^{2}$. The same holds for $\mathcal{F}_{\text {elastic }}^{\varepsilon}$

ii. Since $\operatorname{div} \xi_{E} \in H^{\prime}$, we know that $u$ solves the equation

$$
\int_{\Omega} \nabla u \cdot \nabla u=\int_{\Omega} \xi_{E} \cdot \nabla u
$$

Thus,

and the same holds for $u^{\varepsilon}$.

$$
\|\nabla u\|_{L^{2}}^{2} \leq\|\xi\|_{L^{2}}\|\nabla u\|_{L^{2}}
$$

iii. Note that $u^{\varepsilon}$, as a minimizer of the energy $\int_{\Omega}\left|\nabla u^{\varepsilon}-\xi_{E}^{\varepsilon}\right|^{2}$ is the unique solution of the equation

$$
\triangle u^{\varepsilon}=\operatorname{div} \xi_{E}^{\varepsilon}, \quad u \in H
$$

We can therefore, by the representation theorem, write $u$ as the convolution of the transformation strain with the Green's function for the problem, so that

$$
\begin{aligned}
u^{\varepsilon} & =G * \xi_{E}^{\varepsilon} \\
& =G *\left(\eta^{\varepsilon} * \xi_{E}\right) \\
& =\eta^{\varepsilon} *\left(G * \xi_{E}\right) \\
& =\eta^{\varepsilon} * u,
\end{aligned}
$$


where $u$ is the minimizer of the unmollified elastic energy. From there one can calculate

$$
\begin{aligned}
\left|\nabla u^{\varepsilon}(x)\right| & =\left|\nabla\left(\eta^{\varepsilon} * u\right)(x)\right| \\
& =\left|\int_{\Omega} \nabla_{x} \eta^{\varepsilon}(x-y) u(y) \mathrm{d} y\right| \\
& \leq\left\|\nabla \eta^{\varepsilon}\right\|_{L^{\infty}}\|u\|_{L^{1}} \\
& \leq\left\|\nabla \eta^{\varepsilon}\right\|_{L^{\infty}}\|u\|_{L^{2}} \sqrt{\mathrm{m}(\Omega)} \leq \frac{M}{\varepsilon^{n}}
\end{aligned}
$$

which, after absorbing any additional constant strain stemming from the boundary conditions into $M$, proves the statement ${ }^{1}$.

Next, we will prove an estimate of the change in mollified elastic energy if an area of a certain size changes from one transformation strain to the other. Such a proposition will make it possible to use the aforementioned density lemma for balls of small enough radius.

Lemma 3.2. There exists a uniform constant $M$ such that given two sets of finite perimeter $E_{1}$ and $E_{2}$, we have

$$
\left|\mathcal{F}_{\text {elastic }}^{\varepsilon}\left(E_{1}\right)-\mathcal{F}_{\text {elastic }}^{\varepsilon}\left(E_{2}\right)\right| \leq \frac{M}{\varepsilon^{n}} \cdot \mathrm{m}\left(E_{1} \Delta E_{2}\right) .
$$

Proof. Assume that $\mathcal{F}_{\text {elastic }}^{\varepsilon}\left(E_{1}\right) \leq \mathcal{F}_{\text {elastic }}^{\varepsilon}\left(E_{2}\right)$. Let $A=E_{1} \triangle E_{2}$ and $\xi_{A}$, supported on $A$, such that $\xi_{E_{2}}^{\varepsilon}=\xi_{E_{1}}^{\varepsilon}+\xi_{A}$. Consider the minimizer $u_{1}^{\varepsilon}$ of the energy

$$
\int_{\Omega}\left|\nabla u_{1}^{\varepsilon}-\xi_{E_{1}}^{\varepsilon}\right|^{2}
$$

and use it as a test function for the larger energy. This yields

$$
\begin{aligned}
\mathcal{F}_{\text {elastic }}^{\varepsilon}\left(E_{2}\right)= & \min _{u} \int_{\Omega} \frac{1}{2}\left|\nabla u-\xi_{E_{2}}^{\varepsilon}\right|^{2} \\
\leq & \int_{\Omega} \frac{1}{2}\left|\nabla u_{1}^{\varepsilon}-\xi_{E_{1}}^{\varepsilon}-\xi_{A}\right|^{2} \\
= & \int_{\Omega} \frac{1}{2}\left|\nabla u_{1}^{\varepsilon}-\xi_{E_{1}}^{\varepsilon}\right|^{2} \\
& +\int_{\Omega} \frac{1}{2}\left|\xi_{A}\right|^{2}+\int_{\Omega} 2 \xi_{E_{1}} \cdot \xi_{A}-\int_{\Omega} 2 \nabla u_{1} \cdot \xi_{A} \\
\leq & \mathcal{F}_{\text {elastic }}^{\varepsilon}\left(E_{1}\right) \\
& +\frac{1}{2} \mathrm{~m}(A)\left(\left\|\left.|| \xi_{A}\right|^{2}\right\|_{L^{\infty}}+2\left\|\xi_{E_{1}}\right\|_{L^{\infty}}\left\|\xi_{A}\right\|_{L^{\infty}}\right) \\
& +\operatorname{m}(A)\left\|\xi_{A}\right\|_{L^{\infty}}\left\|\nabla u_{1}^{\varepsilon}\right\|_{L^{\infty}} \\
\leq & \mathcal{F}_{\text {elastic }}^{\varepsilon}\left(E_{1}\right)+\frac{M}{\varepsilon^{n}} \mathrm{~m}(A) .
\end{aligned}
$$

\subsection{The implicit time discretization}

We discretize $(\mathrm{P})$ using an implicit finite time-step scheme, as it was heuristically introduced in the beginning of this section. For $h>0, \varepsilon>0$, consider the energy function

$$
\mathcal{F}^{h, \varepsilon}\left(E, E_{0}\right)=\min _{u^{\varepsilon} \in H} \int_{\Omega}\left|\nabla u^{\varepsilon}-\xi_{E}^{\varepsilon}\right|^{2}+\int_{\Omega}\left|\nabla \chi_{E}\right|+\frac{1}{h} \int_{E \Delta E_{0}} \operatorname{dist}\left(\cdot, \partial E_{0}\right) .
$$

We will call the first term the elastic term, the second term is the perimeter term and the third term is the distance term.

Theorem 3.3. For a given set of finite perimeter $E_{0}$, the set function $\mathcal{F}^{h, \varepsilon}\left(\cdot, E_{0}\right)$ admits a minimizer E. Furthermore, E has finite perimeter.

\footnotetext{
${ }^{1}$ In order for the Green's function to be regular enough to perform this calculation, the boundary of $\Omega$ has to be smooth. One can also refer to the theorems about higher regularity of solutions to elliptic equations, for example in [10], which require a $C^{2}$ boundary.
} 
Proof. The energy $\mathcal{F}^{h, \varepsilon}$ is non-negative, and thus is uniformly bounded from below. Consider therefore a minimizing sequence of sets $\left\{E_{k}\right\}_{k=1}^{\infty}$ for the energy $\mathcal{F}^{h, \varepsilon}$. The elastic energy term in $\mathcal{F}^{h, \varepsilon}$ is uniformly bounded according to Lemma 3.1. The third term, the distance term, is - for a fixed $h$-also uniformly bounded from both above and below. Therefore, one can find a (renumbered) subsequence such that $\int_{\Omega}\left|\nabla \chi_{E_{k}}\right|$, the perimeter of $E_{k}$ is bounded uniformly in $k$. Thus, by Theorem 4.7 and 4.8 in [4] (SBV closedness and compactness), there exists a (renumbered) subsequence, and a set of bounded perimeter $E$, such that

$$
\chi_{E_{k}} \rightarrow \chi_{E} \quad \text { in } L^{1} .
$$

It remains to be shown that $\mathcal{F}^{h, \varepsilon}$ is lower semicontinuous with respect to this convergence. First, by Theorem 4.7 in [4], the perimeter term of $\mathcal{F}^{h, \varepsilon}$ is lower semicontinuous. The strong convergence in $L^{1}$ also immediately implies the continuity of the distance term. For the elastic term, note that the solution operator for the minimization problem of $u^{\varepsilon}$ is continuous with respect to $\xi_{E}^{\varepsilon}$ in $H^{\prime}$. It is therefore clear that the minimizers and minima $u_{i}^{\varepsilon}$ of $\int_{\Omega}\left|\nabla u_{k}^{\varepsilon}-\xi_{E_{k}}^{\varepsilon}\right|^{2}$ converge to a minimizer and minimum of $\int_{\Omega}\left|\nabla u_{k}^{\varepsilon}-\xi_{E}^{\varepsilon}\right|^{2}$. This establishes the lower semicontinuity of $\mathcal{F}^{h, \varepsilon}$.

Given an initial value $E(0)=E_{0}$, and setting $h=\frac{T}{N}, N \in \mathbb{N}$ we can now iteratively, up to $k=N$, define the time-discrete solution to be

$$
E^{h, \varepsilon}(t)= \begin{cases}\operatorname{argmin} \mathcal{F}^{h, \varepsilon}\left(\cdot, E^{h, \varepsilon}(t-h)\right) & \text { if } t=k h \\ E^{h, \varepsilon}((k-1) h) & \text { for } t \in((k-1) h, k h)\end{cases}
$$

as well as

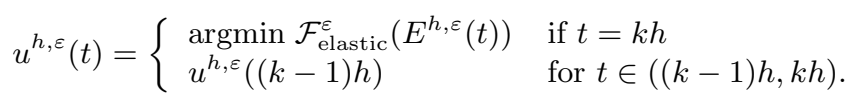

This is the piecewise constant interpolation between the iterated minimizers of the time-step minimization problem. Furthermore, we define for a sequence $\left\{h_{i}\right\}_{i=1}^{\infty}$ such that $h \rightarrow 0$, the sequence of time-discrete solutions $\left\{E^{h_{i}, \varepsilon}(t)\right\}_{i=1}^{\infty}$.

\subsection{Compactness of the time-discrete solution}

We now show that the sequence of time-discrete solutions is strongly equicontinuous in $h$. This enables us to use the Frechet-Kolmogorov theorem to establish strong convergence of a subsequence.

For the rest of this section we will assume the scaling $\varepsilon \geq h^{1 / n}$ and write $\mathcal{F}^{h}\left(E, E_{0}\right):=\mathcal{F}^{h, \varepsilon(h)}\left(E, E_{0}\right)$, as well as $E^{h}(t):=E^{h, \varepsilon(h)}(t)$. The scaling $\varepsilon=h^{1 / n}$ is sufficient for the compactness estimates, but for the estimates in Section 4 we need to choose $\varepsilon=h^{1 / 2 n}$. The constant $C$ is independent of $h$, but may depend on $n, \Omega, T$, and $E_{0}$, and possibly change from line to line.

Compactness in space. The compactness in the spatial variables follows directly from the uniform bound on the perimeter of the transformed set $E$, which we presently prove.

Lemma 3.4. Let $E^{h}(t)$ be the piecewise constant solution constructed above. Then

$$
\int_{\Omega}\left|\nabla \chi_{E^{h}(t)}\right| \leq C
$$

Proof. Let $C=\sup _{\varepsilon>0} \mathcal{F}_{\text {elastic }}^{\varepsilon}\left(E_{0}\right)+\int_{\Omega}\left|\nabla \chi_{E_{0}}\right|<\infty$ by Lemma 3.1. Assume, by contradiction, that for some $h>0$ and some $t<T$, we have $\int_{\Omega}\left|\nabla \chi_{E^{h}(t)}\right|>C$. That means that there must exist a $k \leq N$, such that

$$
\begin{aligned}
& \mathcal{F}_{\text {elastic }}^{\varepsilon}\left(E^{h}(k h)\right)+\int_{\Omega}\left|\nabla \chi_{E^{h}(k h)}\right| \\
> & \mathcal{F}_{\text {elastic }}^{\varepsilon}\left(E^{h}((k-1) h)\right)+\int_{\Omega}\left|\nabla \chi_{E^{h}((k-1) h)}\right|,
\end{aligned}
$$

since

$$
\mathcal{F}_{\text {elastic }}^{h^{1 / 2 n}}\left(E_{0}\right)+\int_{\Omega}\left|\nabla \chi_{E_{0}}\right|<C
$$

This means, however, that $E^{h}(k h)$ can not be a minimizer of the finite time-step problem.

Proposition 1.2 in [11] can now be applied to assert compactness in space. We state this proposition here for the convenience of the reader. 
Proposition 3.5 (Luckhaus-Sturzenhecker). The discrete solution $E^{h}(t)$ fulfills

$$
\int_{0}^{T} \int_{\Omega}\left|\chi_{E^{h}(t)}(x+s e)\right| \mathrm{d} x \mathrm{~d} t \longrightarrow 0 \quad \text { as } s \rightarrow 0
$$

uniformly in $h$ for each unit vector $e \in \mathbb{R}^{n}$.

Compactness in time. We now seek to establish a uniform bound on the total variation of the transformed set $E(t)$ in time. The major difficulty here is the inability of the distance term in the energy $\mathcal{F}^{h}$ to yield such a bound by itself. If one were to assume, for example, a rate-independent evolution model, the distance term would be replaced by a penalization of the area $E_{0} \Delta E$ via a term of the form

$$
\mathrm{m}\left(E_{0} \triangle E\right) .
$$

Such an evolution law therefore automatically yields a uniform bound on the total variation of $E(t)$, which then leads to the necessary strong compactness.

In our case, however, since one can find a dense set $E_{\text {bad }}$ in $\Omega$ with arbitrarily low elastic and surface energy, the situation is more complicated. For $E_{0}=E_{\mathrm{bad}}$, no matter what the set in the next timestep looks like, the distance term vanishes. This way, one can generate a sequence of faster and faster oscillating phases on a large part of the domain. Such a sequence will certainly not converge strongly. It is the goal now to exclude such bad sets a priori through some assertions on the geometric properties of sets that minimize the time-step energy $\mathcal{F}^{h}$. We thus in the following extend the density estimate-Lemma 1.3 in [11] to include the elastic energy term.

Lemma 3.6 ( $\mathscr{L}^{n}$-density of minimizing sets). Let $f \in L^{\infty}(\Omega)$ and assume $E$ minimizes the functional

$$
\mathcal{F}(E)=\mathcal{F}_{\text {elastic }}^{\varepsilon}(E)+\int_{\Omega} f \chi_{E}+\int_{\Omega}\left|\nabla \chi_{E}\right|
$$

among all measurable subsets of $\Omega$. Then the density estimate,

$$
\theta \leq f_{B_{\rho}(x)} \chi_{E} \leq 1-\theta
$$

holds for all $x \in \partial E$, such that $\operatorname{dist}(x, \partial \Omega)>\rho$, and for all $0<\rho<\frac{\gamma_{n}}{2\left(\|f\|_{L^{\infty}}+\frac{M}{\varepsilon^{n}}\right) \omega_{n}^{1 / n}}:=\rho_{0}$ such that $B_{\rho}(x) \subset \Omega$. The constant $M$ is the constant from Lemma 3.2 and $\theta$ is given by $\theta=\left(\frac{1}{4}\right)^{n}$. The constant $\omega_{n}$ is the $\mathscr{L}^{1}$ measure of the unit sphere in $\mathbb{R}^{n}$; the constant $\gamma_{n}$ is given by $\gamma_{n}=n \omega_{n}^{1 / n}$.

For $x \in E$ and $x \in E^{c}$, one has, respectively,

$$
\theta \leq f_{B_{\rho}(x)} \chi_{E}
$$

and

$$
\theta \leq f_{B_{\rho}(x)} \chi_{E^{c}}
$$

Furthermore, if $f \geq 0$ in $B_{\rho}(x)$, one has

$$
\theta \leq f_{B_{\rho}(x)} \chi_{E}
$$

for $0<\rho<\frac{\gamma_{n} \varepsilon^{n}}{2 M \omega_{n}^{1 / n}}$, and for $f \leq 0$ the equivalent estimate holds for $E^{c}$. If, above that, one has that $f \geq \frac{M}{\varepsilon^{n}}$ in $B_{\rho}(x)$, the bound on the radius can be dropped entirely on the estimate for $E$.

Proof. We only show the lower density bound; the upper bound can be proved by considering the set $E^{c}$. First we show that for $\rho<\rho_{0}$ and $x$ as above the set $E \cap B_{\rho}(x)$ contains a part of the boundary of $B_{\rho}(x)$, i.e.,

$$
\int_{\partial B_{\rho}(x)} \chi_{E} \mathrm{~d} \mathcal{H}^{n-1}>0
$$

The excluded situation as well as the possible behavior are illustrated in Figure 2. If that does not hold for some $r \in(0, \rho)$, we conclude from the isoperimetric inequality $\int_{\mathbb{R}^{n}}\left|\nabla \chi_{E}\right| \geq \gamma_{n}\left(\int_{\mathbb{R}^{n}} \chi_{E}\right)^{\frac{n-1}{n}}$ that

$$
\gamma_{n}\left|\int_{B_{r}(x)} \chi_{E}\right|^{\frac{n-1}{n}} \leq \int_{B_{r}(x)}\left|\nabla \chi_{E}\right| .
$$



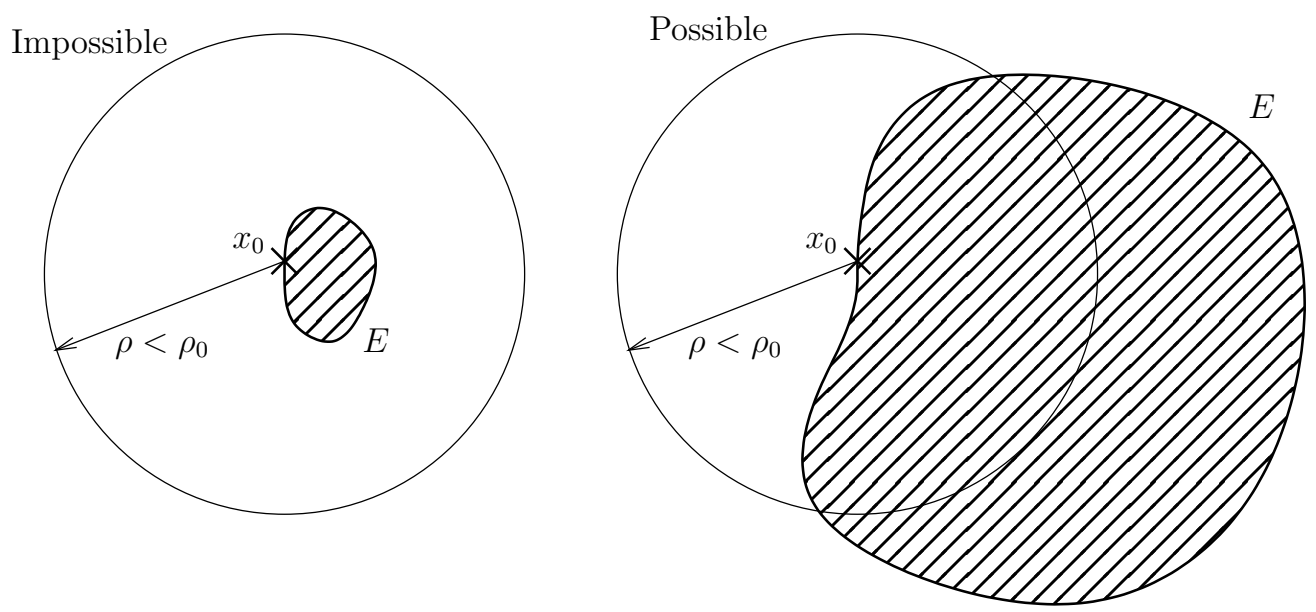

Figure 2: For a ball centered on the boundary of the set $E$ with small enough radius, the set $E$ actually has to intersect the boundary of the ball. The situation on the left is therefore excluded, a possible minimizing set is shown on the right for comparison.

Further, the minimality of the set $E$ requires

$$
\begin{aligned}
& \int_{B_{r}(x)}\left|\nabla \chi_{E}\right| \\
\leq & \left(\|f\|_{L^{\infty}}+\frac{M}{\varepsilon^{n}}\right) \int_{B_{r}(x)} \chi_{E} \\
= & \left(\|f\|_{L^{\infty}}+\frac{M}{\varepsilon^{n}}\right) \mathrm{m}\left(\chi_{E} \cap B_{r}(x)\right)
\end{aligned}
$$

by comparing the energies of the sets $E$ and the set $E \backslash B_{r}(x)$. Therefore

$$
\omega_{n} \rho^{n} \geq \int_{B_{r}(x)} \chi_{E} \geq\left(\frac{\gamma_{n}}{\|f\|_{L^{\infty}}+\frac{M}{\varepsilon^{n}}}\right)^{n},
$$

which contradicts the upper bound for $\rho$ given above.

In the second step we define, for a fixed $\sigma \in\left(0, \frac{\rho}{2}\right)$, the comparison set

$$
E^{\prime}=E \backslash\left(B_{\frac{\rho}{2}+\sigma}(x) \backslash B_{\frac{\rho}{2}-\sigma}(x)\right) .
$$

For the volume $V(\sigma):=\mathrm{m}\left(E \cap\left(B_{\frac{\rho}{2}+\sigma}(x) \backslash B_{\frac{\rho}{2}-\sigma}(x)\right)\right.$, using again the isoperimetric inequality and the minimality of $E$, we find

$$
\begin{gathered}
\gamma_{n} V(\sigma)^{\frac{n-1}{n}}-A^{+}\left(\frac{\rho}{2}-\sigma\right)-A^{-}\left(\frac{\rho}{2}+\sigma\right) \\
\leq A^{-}\left(\frac{\rho}{2}-\sigma\right)+A^{+}\left(\frac{\rho}{2}+\sigma\right)+\left(\|f\|_{L^{\infty}}+\frac{M}{\varepsilon^{n}}\right) V(\sigma) .
\end{gathered}
$$

Here $A^{+}(r), A^{-}(r)$ denote the sectional surfaces of $E$ with the sphere $\partial B_{r}(x)$, defined by the outer and inner trace of $\chi_{E}$ on $\partial B_{r}(x)$, respectively. Taking the derivative of the volume of the set in the sense of measures shows that, for almost all $\sigma$,

$$
\frac{\mathrm{d}}{\mathrm{d} \sigma} V(\sigma)=A^{+}\left(\frac{\rho}{2}-\sigma\right)+A^{-}\left(\frac{\rho}{2}+\sigma\right)=A^{-}\left(\frac{\rho}{2}-\sigma\right)+A^{+}\left(\frac{\rho}{2}+\sigma\right) .
$$

Therefore we get the differential inequality,

$$
\frac{\mathrm{d}}{\mathrm{d} \sigma} V(\sigma) \geq \frac{\gamma_{n}}{4} V(\sigma)^{\frac{n-1}{n}},
$$




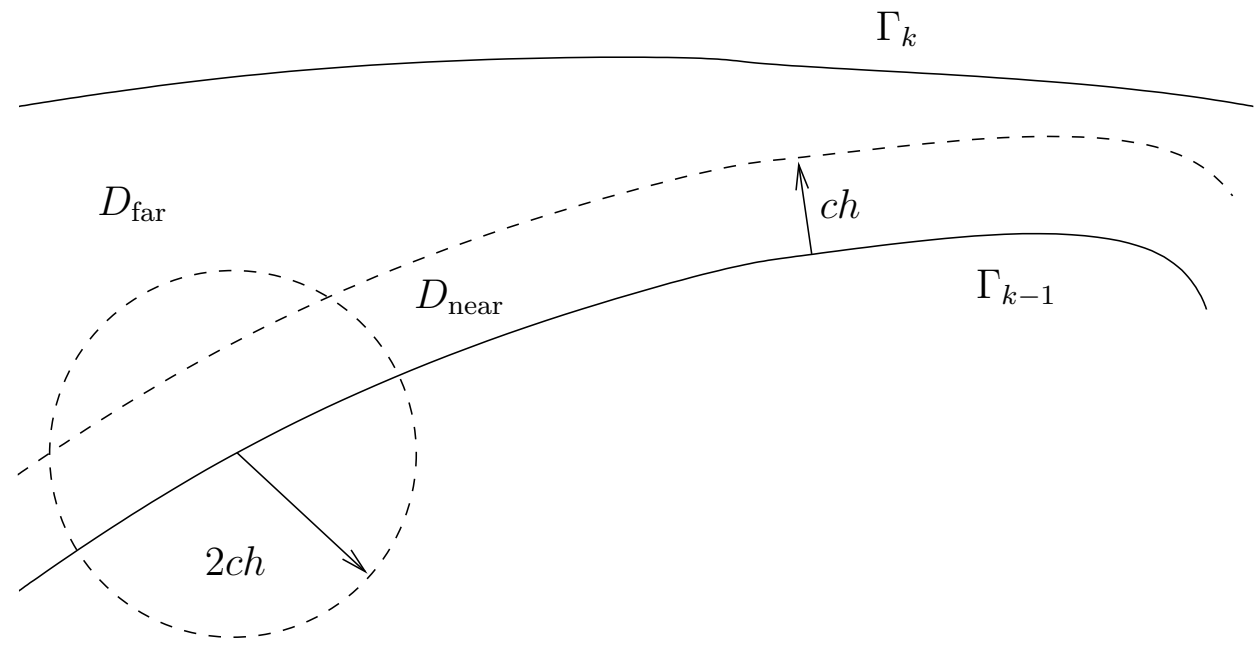

Figure 3: Illustration of Lemma 3.7

where we used that due to the bound on $\rho$,

$$
V(\sigma) \leq\left(\frac{\gamma_{n}}{2\left(\|f\|_{L^{\infty}}+\frac{M}{\varepsilon^{n}}\right)}\right)^{n} .
$$

Integration of this equation yields

$$
\int_{B_{\rho}(x)} \chi_{E} \geq\left(\frac{\gamma_{n}}{4 n}\right)^{n} \rho^{n}
$$

which is the assertion with $\theta=\frac{1}{\omega_{n}}\left(\frac{\gamma_{n}}{4 n}\right)^{n}=1 / 4^{n}$.

The further assertions can be proved by changing the corresponding terms in (45).

Lemma 3.7 (Bound on the variation in time of $\chi_{E}^{h}(t)$ ). There is a constant $C \in \mathbb{R}$, independent of $h$, such that the sets $E_{k}=E^{h}(k h)$, for $k \leq N$ satisfy

$$
\sum_{k=1}^{N} \int_{\Omega}\left|\chi_{E_{k}}-\chi_{E_{k-1}}\right|<C
$$

Proof. We will prove this relation for $E_{k} \backslash E_{k-1}$, the estimate for $E_{k-1} \backslash E_{k}$ follows by a similar procedure. The proof is illustrated in Figure 3.

First, we split the set

$$
\begin{aligned}
E_{k} \backslash E_{k-1} \subset & \left\{x \in E_{k} \backslash E_{k-1}: \operatorname{dist}\left(x, \partial E_{k-1}\right)>c h\right\} \\
& \cup\left\{x \in E_{k} \backslash E_{k-1}: \operatorname{dist}\left(x, \partial E_{k-1}\right) \leq c h\right\} \\
=: & D_{\text {far }} \cup D_{\text {near }} .
\end{aligned}
$$

The constant $c$ is chosen such that

$$
c<\frac{\gamma_{n}}{2 \omega_{n}^{1 / n}} \frac{1}{\operatorname{diam}(\Omega)+M}
$$

which means that the energy $\mathcal{F}^{h}\left(E, E_{0}\right)$ satisfies the conditions of the density estimate for balls of radius $2 c h$, because of the used scaling $\varepsilon \geq h^{1 / n}$.

For the first term in (52), we use the distance term in $\mathcal{F}$ to obtain

$$
\begin{aligned}
\mid\{x & \left.\in E_{k} \backslash E_{k-1}: \operatorname{dist}\left(x, \partial E_{k-1}\right)>c h\right\} \mid<\frac{1}{c h} \int_{E_{k} \Delta E_{k-1}} \operatorname{dist}\left(x, \partial E_{k-1}\right) \mathrm{d} x \\
& \leq \frac{1}{c}\left(\int_{\Omega}\left|\nabla \chi_{E_{k-1}}\right|-\int_{\Omega}\left|\nabla \chi_{E_{k}}\right|+\mathcal{F}_{\text {elastic }}^{\varepsilon^{1 / n}}\left(E_{k-1}\right)-\mathcal{F}_{\text {elastic }}^{\varepsilon^{1 / n}}\left(E_{k}\right)\right) .
\end{aligned}
$$


The second set is estimated by means of the density lemma. We can cover it, up to a distance of $4 c h$ to the boundary of $\Omega$, with a family of balls $B \in \mathscr{B}$ with radius $\rho=2$ ch, all completely contained in $\Omega$, with center $x \in \partial E_{k-1}$, such that the maximum number of balls containing one point is bounded by a constant $D(n)$. Employing the density lemma and an isoperimetric inequality in the interior of a ball, we obtain

$$
\int_{B} \chi_{E_{k} \backslash E_{k-1}} \leq \omega_{n} \rho^{n} \leq c \rho \int_{B}\left|\nabla \chi_{E_{k-1}}\right|
$$

for each $B$. Summation over all $B \in \mathscr{B}$ and adding the boundary term yields

$$
\left|\left\{x \in E_{k} \backslash E_{k-1}: \operatorname{dist}\left(x, \partial E_{k-1}\right) \leq c h\right\}\right| \leq D(n) C c h \int_{\Omega}\left|\nabla \chi_{E_{k-1}}\right|+C h .
$$

From (55) and (57) we get

$$
\begin{aligned}
& \sum_{k=1}^{N} \mathrm{~m}\left(E_{k} \backslash E_{k-1}\right) \\
\leq & \sum_{k=1}^{N} \frac{1}{c}\left(\int_{\Omega}\left|\nabla \chi_{E_{k-1}}\right|-\int_{\Omega}\left|\nabla \chi_{E_{k}}\right|+\mathcal{F}_{\text {elastic }}^{\varepsilon^{1 / n}}\left(E_{k-1}\right)-\mathcal{F}_{\text {elastic }}^{\varepsilon^{1 / n}}\left(E_{k}\right)\right) \\
& +\sum_{k=2}^{N} D(n) C c h \int_{\Omega}\left|\nabla \chi_{E_{k-1}}\right|+C+\mathrm{m}(\Omega) \\
< & C .
\end{aligned}
$$

Using the estimate (58), compactness in time now follows from Proposition 1.6 in [11].

Proposition 3.8 (Luckhaus-Sturzenhecker). We have that

$$
\int_{0}^{t-\tau} \int_{\Omega}\left|\chi_{E^{h}(t+\tau)}(x)\right| \mathrm{d} x \mathrm{~d} t<c \tau .
$$

Convergence of the discrete solution The previous compactness considerations lead to the following:

Theorem 3.9 (Convergence). The sequence $\chi_{E^{h}(t)}$ contains a subsequence, converging strongly in $L^{1}\left(\Omega_{T}\right)$ and weakly in $B V\left(\Omega_{T}\right)$, with a limit function

$$
\chi: \Omega_{T} \rightarrow\{0,1\} \in L^{\infty}((0, T) ; B V(\Omega)) .
$$

Additionally, we have

$$
u^{h} \rightarrow u \in L^{2}((0, T) ; H(\Omega))
$$

strongly in $L^{2}((0, T) ; H(\Omega))$ for the same subsequence.

Proof. Compactness in space and compactness in time leads to the applicability of the Frechet-Kolmogorov theorem for the sequence $\chi_{E^{h}}$, so the convergence of the characteristic functions is strong. Let $E$ be the set $\left\{x \in \Omega_{T}\right.$ such that $\left.\chi(x)=1\right\}$ corresponding to the limiting characteristic function. We then also have that

$$
\xi_{E^{h}}^{\varepsilon} \rightarrow \xi_{E}
$$

strongly in $L^{2}\left((0, T) ; L^{2}(\Omega)\right)$. Denote by $\mathcal{T}$ the operator that maps a function $\xi(\cdot, t)$ in $L^{2}$ to the weak solution of $\triangle u(\cdot, t)=\operatorname{div} \xi(\cdot, t)$. This is a continuous linear operator from $L^{2}(\Omega)$ to $H(\Omega)$. It follows that the operator

$$
\mathcal{S}: L^{2}\left((0, T) ; L^{2}(\Omega)\right) \rightarrow L^{2}((0, T) ; H(\Omega))
$$

defined by applying $\mathcal{T}$ at every time $t \in(0, T)$ is also linear and continuous. We therefore have strong convergence of $u$, due to the strong convergence of the right hand side.

Remark 3.10. Note that this ensures also that $\nabla \chi_{E^{h}} \rightarrow \nabla \chi$ in $\operatorname{rca}\left(\Omega_{T}\right)$. 


\section{The limit problem}

In the previous section is was proved that the sequence of discrete solutions contains a strongly converging subsequence. The task in this section is to prove that the limiting function is a solution to Problem (P). It is necessary, however, to require that, in the limit, there is no loss of surface area. This ensures the convergence of $|\nabla \chi|$ in the sense of radon measures. We thus from now on assume that

$$
\int_{\Omega_{T}}\left|\nabla \chi_{E^{h}}\right| \rightarrow \int_{\Omega_{T}}|\nabla \chi|
$$

As in [11], we define the discrete normal velocity $v_{n}^{h}(x)=\frac{1}{h} \operatorname{sdist}\left(x, \partial E^{h}(t-h)\right)$, and it is necessary to prove some bounds on it, in order to show its convergence to a suitable $v_{n}$ in the limiting equation. For the remainder of this section we will assume the scaling $\varepsilon=h^{1 / 2 n}$.

Lemma 4.1 (Estimates on $v_{n}^{h}$ ). There exists $c \in \mathbb{R}$ such that we have i) $\left\|v_{n}^{h}\right\|_{L^{\infty}\left(E^{h}(t) \Delta E^{h}(t-h)\right)}<c h^{-1 / 2}$

ii) $\int_{\left.\left\{|| v_{n}^{h}||>Q\right\} \cup \Omega_{T}\right\}}\left|v_{n}^{h}\right|\left|\nabla \chi_{E^{h}}\right|<\frac{c}{Q}$

iii) $\int_{\Omega_{T}}\left(v_{n}^{h}\right)^{2}\left|\nabla \chi_{E^{h}}\right|<c$.

Proof. i) Let $x \in E^{h}(t) \backslash E^{h}(t-h)$ such that

$$
\left|v_{n}^{h}(x)\right|=\frac{1}{h} \operatorname{dist}\left(x, \partial E^{h}(t-h)\right) \geq c h^{-1 / 2}
$$

for some $t$. The case of $x \in E^{h}(t-h) \backslash E^{h}(t)$ can be treated analogously. We compare the energy of the set $E^{h}(t)$ with the set $E^{\prime}=E^{h}(t) \backslash B_{\frac{1}{2} c h^{1 / 2}}(x)$, where a ball of radius $\frac{1}{2} \operatorname{ch}^{1 / 2}$ around $x$ has been cut out. In order for the set $E_{t}$ to be energy minimizing, the additional surface energy that such a ball would cause must be larger than the gain in the distance and the elastic part of the energy. We get

$$
n \omega_{n}\left(\frac{1}{2} c h^{1 / 2}\right)^{n-1} \geq\left(\frac{1}{2} c h^{-1 / 2}-M h^{-1 / 2}\right) \int_{B_{\frac{1}{2} c h^{1 / 2}}(x)} \chi_{E^{h}(t)} .
$$

For $c$ sufficiently large, we have $c h^{-1 / 2}>M h^{-1 / 2}$, and the restriction on the size of the balls in the density estimate can be dropped. We therefore get a lower bound for $\int_{B_{\frac{1}{2} c h^{1 / 2}(x)}} \chi_{E^{h}(t)}$, which leads to

$$
n \omega_{n}\left(\frac{1}{2} c h^{1 / 2}\right)^{n-1} \geq\left(\frac{1}{2} c h^{-1 / 2}-M h^{-1 / 2}\right) \theta \omega_{n}\left(\frac{1}{2} c h^{1 / 2}\right)^{n},
$$

which, for a possibly even larger $c$, yields a contradiction.

ii) From i) above, we have that $\int_{\left.\left\{|| v_{n}^{h}||>Q\right\} \cup \Omega_{T}\right\}}\left|v_{n}^{h}\right|\left|\nabla \chi_{E^{h}}\right|=0$ unless $Q \leq c h^{1 / 2}$, we can therefore assume that $h$ satisfies this relation. Pick $\tilde{c}$ such that $\frac{\tilde{c}}{2} Q h \leq \frac{\tilde{c}}{2 \sqrt{c}} h^{1 / 2}<\frac{\gamma_{n} \varepsilon^{n}}{2 M \omega_{n}^{1 / n}}$, the constant from the density estimate, noting that $f \geq 0$ in the region we are considering here.

Consider now, for $Q>0$, the set

$$
F=\partial E^{h}(t) \cap\left\{2^{l} Q<\left|v_{n}^{h}\right| \leq 2^{l+1} Q\right\} \text { for a fixed } t=k h ; k, l \in \mathbb{N}
$$

and a ball $B=B_{\frac{\tilde{c}}{2} Q h}(x), x \in F$. By applying the density estimate, we obtain

$$
\int_{B \cap\left(E^{h}(t) \Delta E^{h}(t-h)\right)}\left|v_{n}^{h}\right|>2^{l} c Q^{n+1} h^{n} .
$$

The energy minimizing property of $E_{t}$, however, requires that

$$
\int_{B}\left|v_{n}^{h}\right|\left|\nabla \chi_{E^{h}(t)}\right|<2^{l+1} c^{\prime} Q^{n} h^{n-1}+h^{n} Q^{n} \frac{M}{\sqrt{h}}<2^{l+1} c^{\prime} Q^{n} h^{n-1} .
$$

For the second inequality, one has to require $h$ to be small enough. From here on one can continue as in the proof of Lemma 2.1 in [11], and the proof of iii) also follows along the that line. 
With these estimates at hand, it is possible to employ Proposition 2.2 from [11] to ensure that the error in the discrete velocity vanishes.

Proposition 4.2 (Luckhaus-Sturzenhecker). If $n<7$, the error estimate in the discrete curvature equation vanishes as $h \rightarrow 0$, i.e,

$$
\int_{\Omega_{T}}\left(\partial_{t}^{-h} \chi_{E^{h}}-\frac{1}{h} \operatorname{sdist}\left(\cdot, \partial^{h}(t-h)\right)\left|\nabla \chi_{E^{h}}\right|\right) \eta \longrightarrow 0
$$

as $h \rightarrow 0$ for all $\eta \in C_{0}^{0}\left(\Omega_{T}, \mathbb{R}\right)$.

The symbol $\partial_{t}^{-h}$ denotes the discrete backward time derivative with time-step $h$.

Now it is possible to state the conditional existence theorem.

Theorem 4.3 (Existence). Let $n \leq 6$. If the time discrete solution satisfies $\int_{\Omega_{T}}\left|\nabla \chi_{E^{h}}\right| \rightarrow \int_{\Omega_{T}}|\nabla \chi|$, then there exist functions $\chi: \Omega_{T} \rightarrow\{0,1\}, v_{n}: \Omega_{T} \rightarrow \mathbb{R}$, and $u: \Omega_{T} \rightarrow \mathbb{R}$ that solve Problem $(P)$.

Proof. Noting that, for a fixed $t=h l, l \in \mathbb{N}$, the minimizer of the time discrete problem, and thus the time discrete solution, satisfies the weak equations

$$
\begin{aligned}
& \int_{\Omega}\left(\operatorname{div} \zeta-\frac{\nabla \chi}{|\nabla \chi|} \nabla \zeta \frac{\nabla \chi}{|\nabla \chi|}\right)|\nabla \chi|+ \\
& \int_{\Omega}(W \cdot I d-\nabla u \otimes \sigma) \nabla \zeta+\int_{\Omega} \frac{1}{h} \operatorname{sdist}\left(\cdot, \partial E_{t-h}\right) \zeta \nabla \chi=0
\end{aligned}
$$

for $\zeta \in C_{0}^{\infty}\left(\Omega, \mathbb{R}^{n}\right)$ and

$$
\int_{\Omega_{T}}\left(\nabla u-\xi_{E}\right) \nabla \phi=0
$$

for $\phi \in H^{\prime}(\Omega)$, one can apply the proof of Theorem 2.3 in [11]. The convergence of the time discrete solution of the elliptic problem to a solution of the limit problem is ensured by the strong convergence established by Proposition 3.9.

Acknowledgment This work draws from the doctoral thesis of Patrick Dondl at the California Institute of Technology. We gratefully acknowledge the financial support of the National Science Foundation (ACI-0204932, DMS-0311788).

\section{References}

[1] R. Abeyaratne and J. K. Knowles. On the driving traction acting on a surface of strain discontinuity in a continuum. J. Mech. Phys. Solids, 38(3):345-360, 1990.

[2] R. Abeyaratne and J. K. Knowles. Kinetic relations and the propagation of phase boundaries in solids. Arch. Rational Mech. Anal., 114(2):119-154, 1991.

[3] F. Almgren, J. E. Taylor, and L. Wang. Curvature-driven flows: a variational approach. SIAM J. Control Optim., 31(2):387-438, 1993.

[4] L. Ambrosio, N. Fusco, and D. Pallara. Functions of bounded variation and free discontinuity problems. Oxford Mathematical Monographs. Oxford University Press, 2000.

[5] L. Ambrosio, N. Gigli, and G. Savaré. Gradient flows in metric spaces and in the space of probability measures. Lectures in Mathematics ETH Zürich. Birkhäuser Verlag, 2005.

[6] J. M. Ball and R. D. James. Fine phase mixtures as minimizers of energy. Arch. Rational Mech. Anal., 100(1):13-52, 1987.

[7] K. Bhattacharya. Microstructure of Martensite: Why It Forms and How It Gives Rise to the ShapeMemory Effect. Oxford University Press, 2003.

[8] P. W. Dondl and Kaushik Bhattacharya. Pinning and depinning of martensitic interfaces in the shallow slope approximation. In preparation.

[9] J. L. Ericksen. Equilibrium of bars. J. Elasticity, 5(3-4):191-201, 1975. Special issue dedicated to A. E. Green.

[10] L. C. Evans. Partial differential equations, volume 19 of Graduate Studies in Mathematics. American Mathematical Society, 1998. 
[11] S. Luckhaus and T. Sturzenhecker. Implicit time discretization for the mean curvature flow equation. Calc. Var. Partial Differential Equations, 3(2):253-271, 1995.

[12] A. Mielke. Evolution of rate-independent systems. In Evolutionary equations. Vol. II, Handb. Differ. Equ., pages 461-559. Elsevier/North-Holland, Amsterdam, 2005. 